\title{
THE DEMAND TENDENCIES IN ECONOMIC EDUCATION AND CURRENT POLICY CHALLENGES
}

\section{ANA GVRITISHVILI}

\section{PhD student}

Ivane Javakhishvili Tbilisi state university, Georgia

ana.gvritishvili@tsu.ge

Abstract. The purpose of this work is to demonstrate the importance of economic education for sustainable development of the country. Also, to study the tendencies of its demand in Georgia. The study analyzes a number of entrants willing to enroll in the Bachelor and Master degree economic programmes for 2013-2018 years. Also, it analyzes the average dynamics of scores of applicants in the same years. Analysis showed that the number of entrants, as well as graduates, selecting the profession of economics, is decreasing according to years. Furthermore, this analysis allows us to assume that the financing of the economic programme is less likely to determine the choice of applicants.

KEYWORDS: ECONOMIC EDUCATION, ENTRANT, DEMAND ON ECONOMIC EDUCATION.

For citation: Gvritishvili, A. (2020). The Demand tendencies in economic education and current policy challenges. Globalization and Business, №9, pp. 187-193 (In Georgian). https://doi.org/10.35945/gb.2020.09.023 


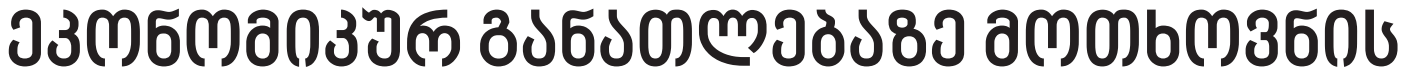

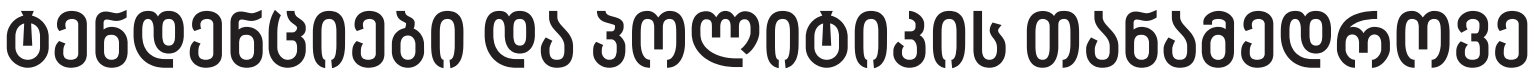 зЈวल63030วั0
}

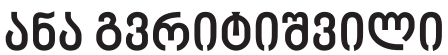

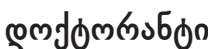

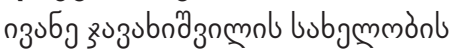

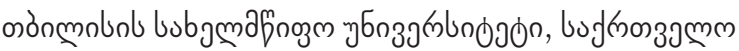 \\ ana.gvritishvili@tsu.ge
}

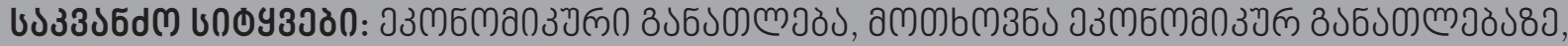

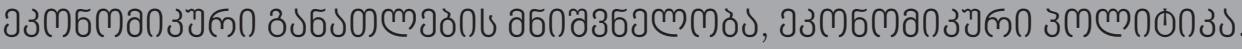

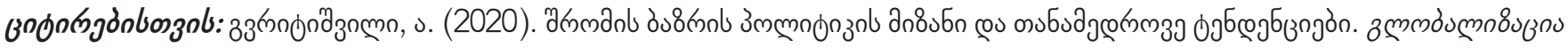
sou do86glon, №9, 33. 187-193. https://doi.org/10.35945/gb.2020.09.023

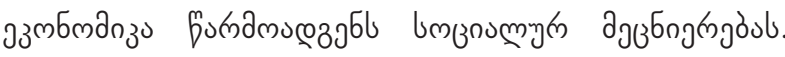

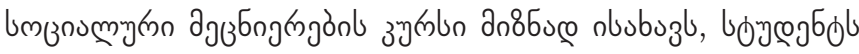

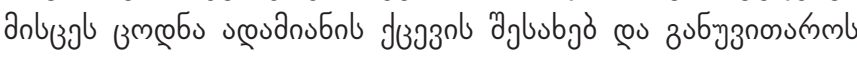

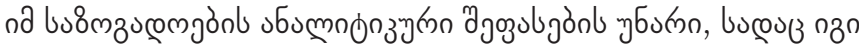

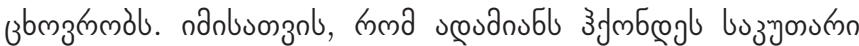

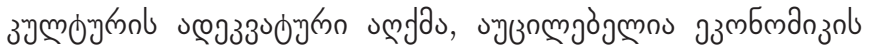

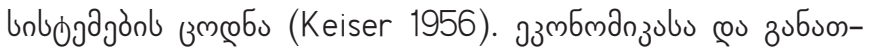

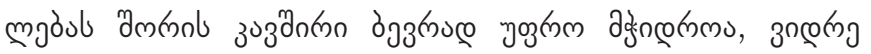

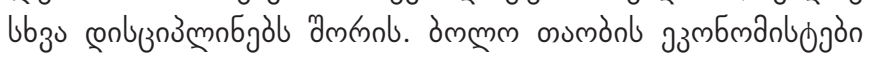

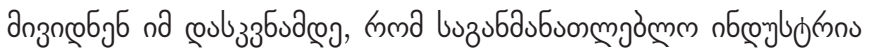

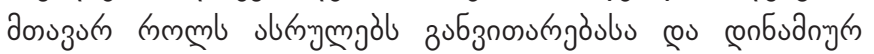

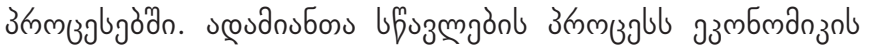

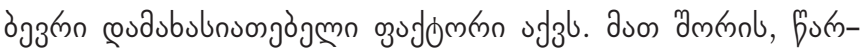

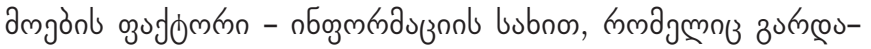
пуаб jös змкобuco (Boulding 1969).

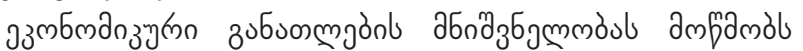

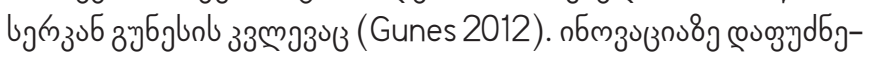

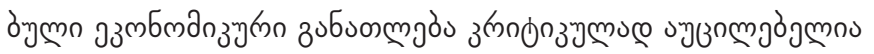

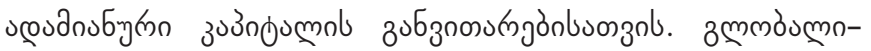

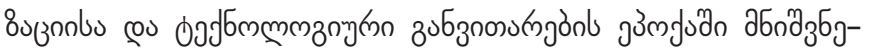

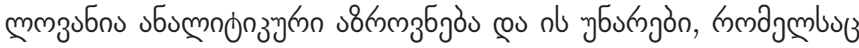

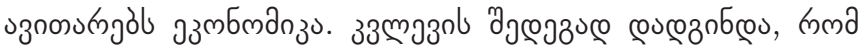

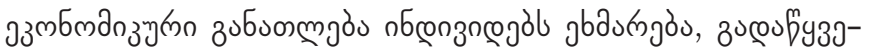

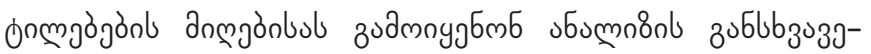

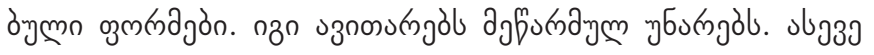

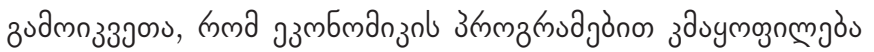

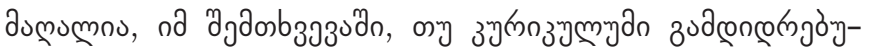

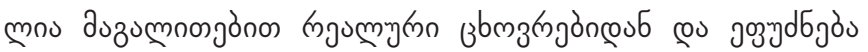

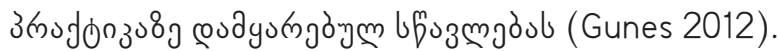

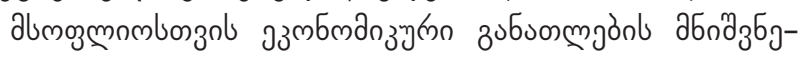

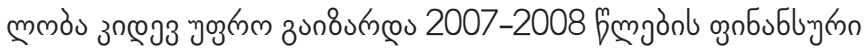

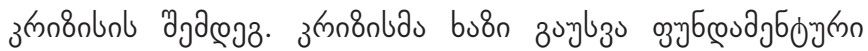

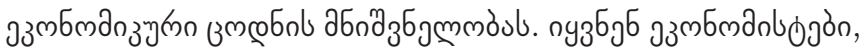

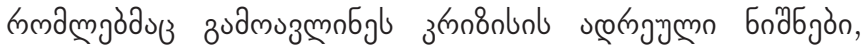

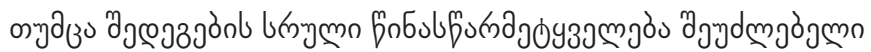

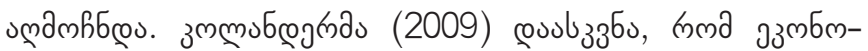

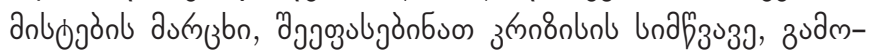

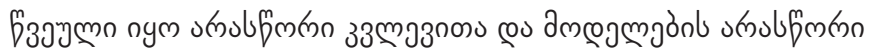

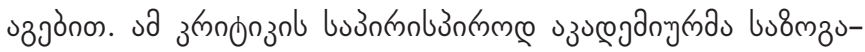

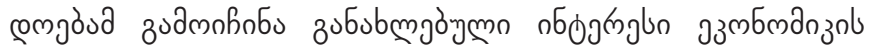

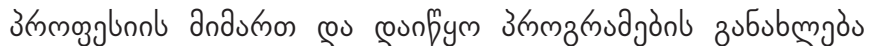

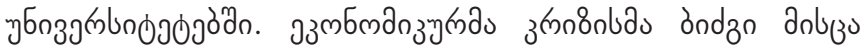

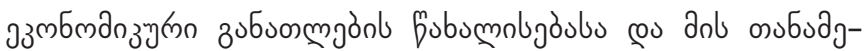

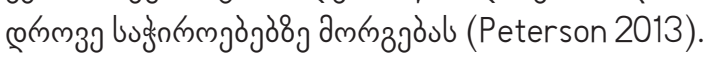

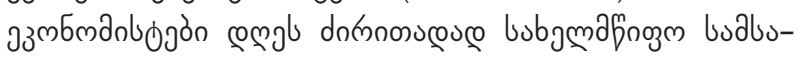

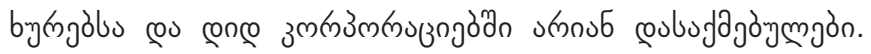

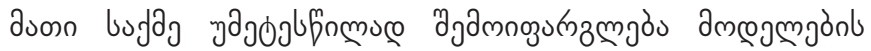

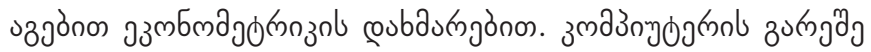

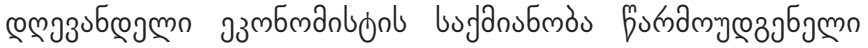

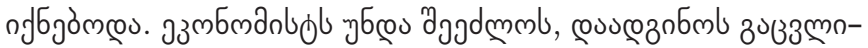

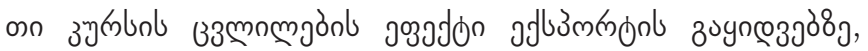

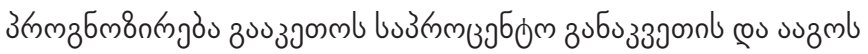

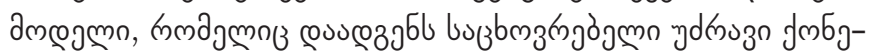
on bu ojubgal (Robinson and Davis 1999).

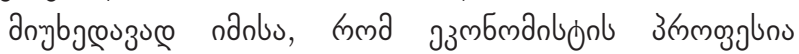

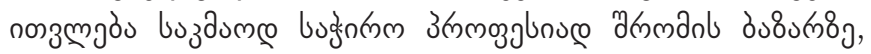




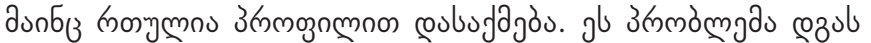

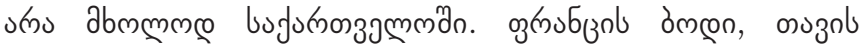

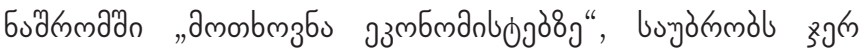

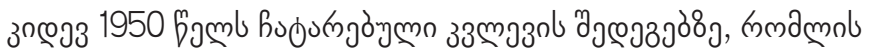

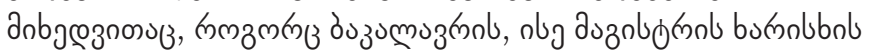

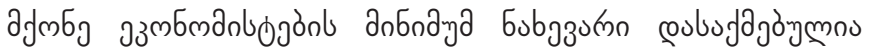

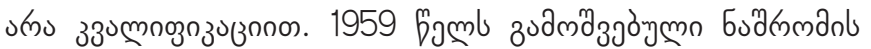

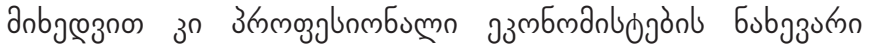

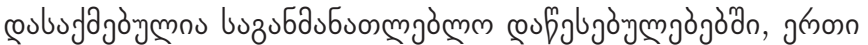

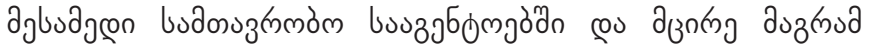

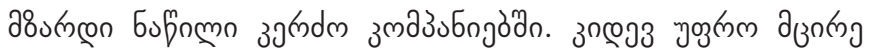

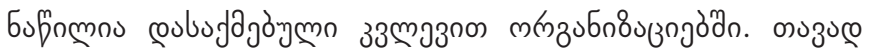

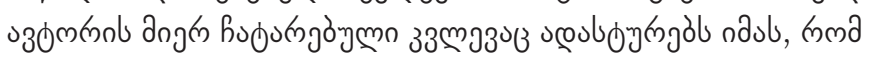

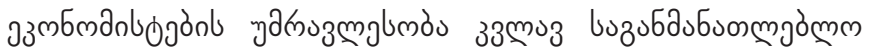

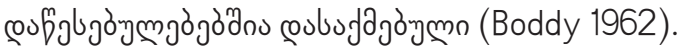

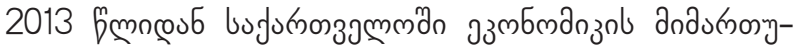

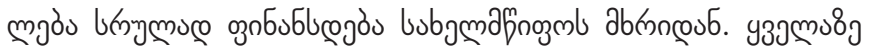

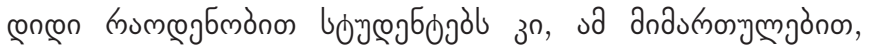

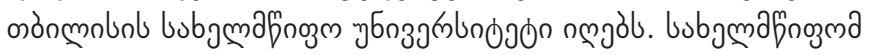

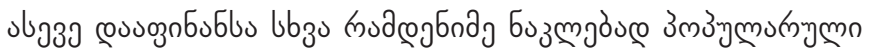

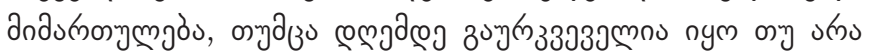

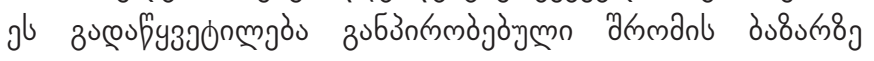

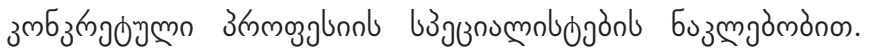

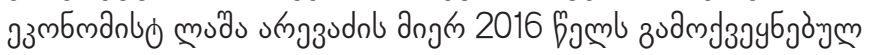

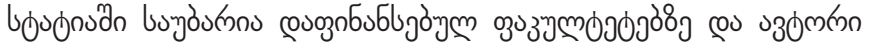

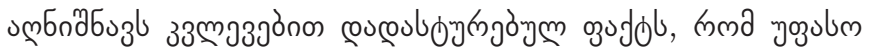

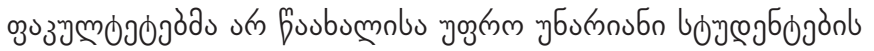

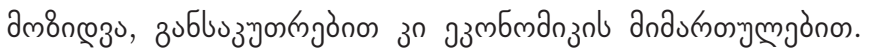

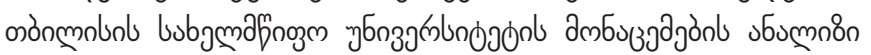

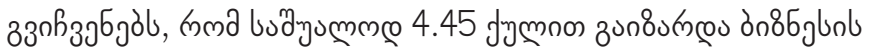

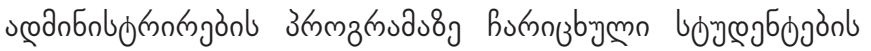

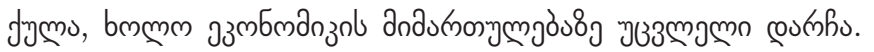

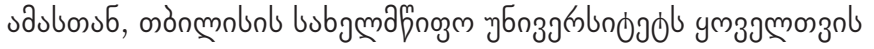

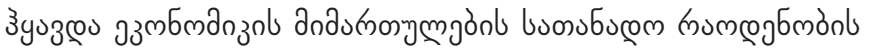
змбопзэбол (Arevadze, 2016).

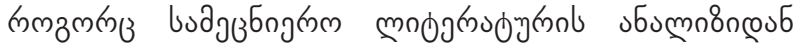

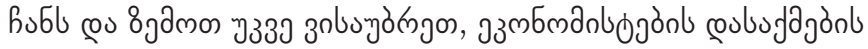

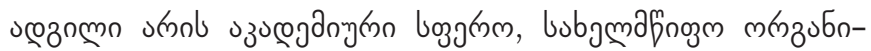

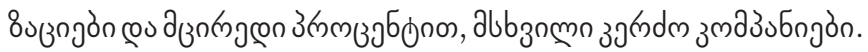

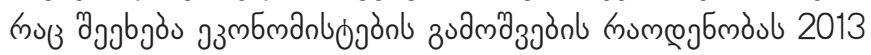

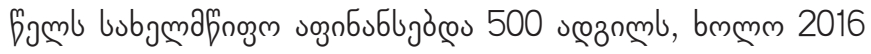

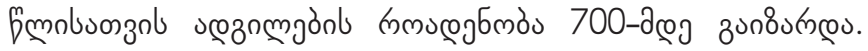

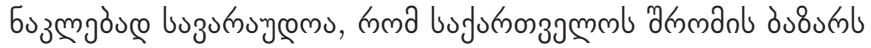

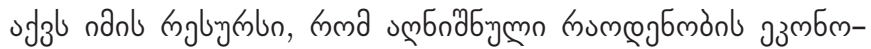
anbojón çubufaç

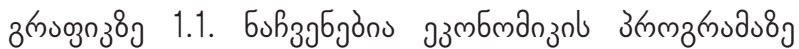

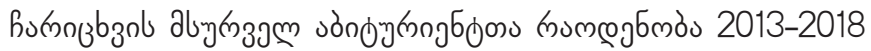

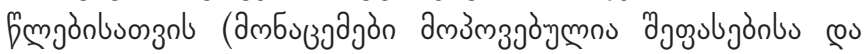

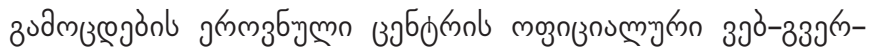

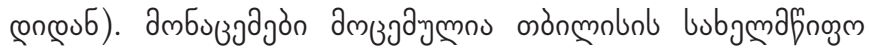

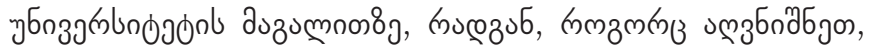

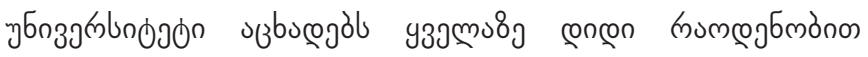

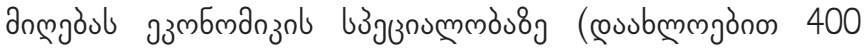
sòn

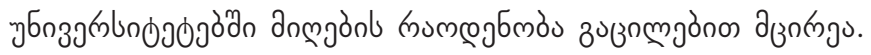

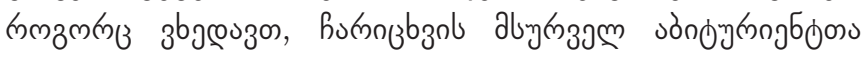

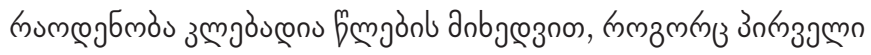

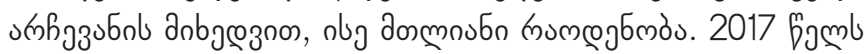

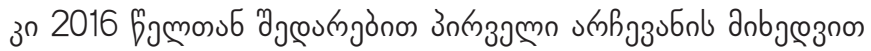

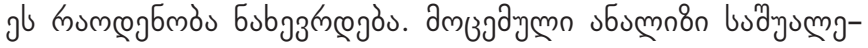

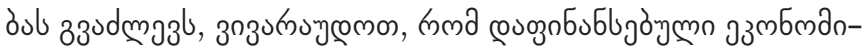

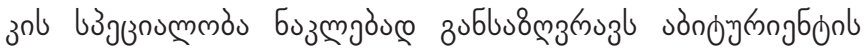
sरhozubl.

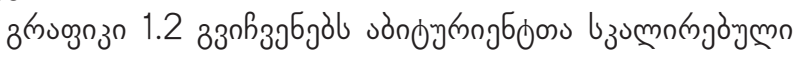

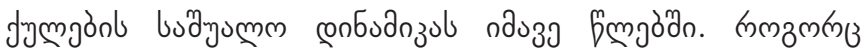

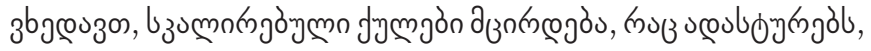

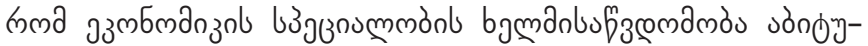

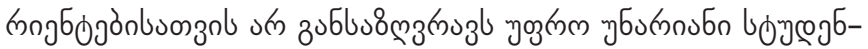

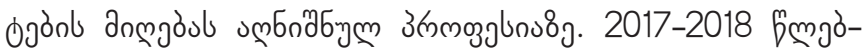

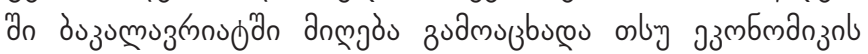

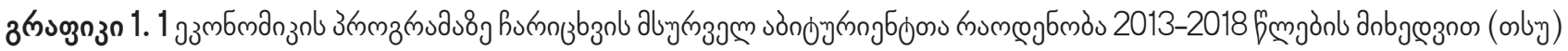

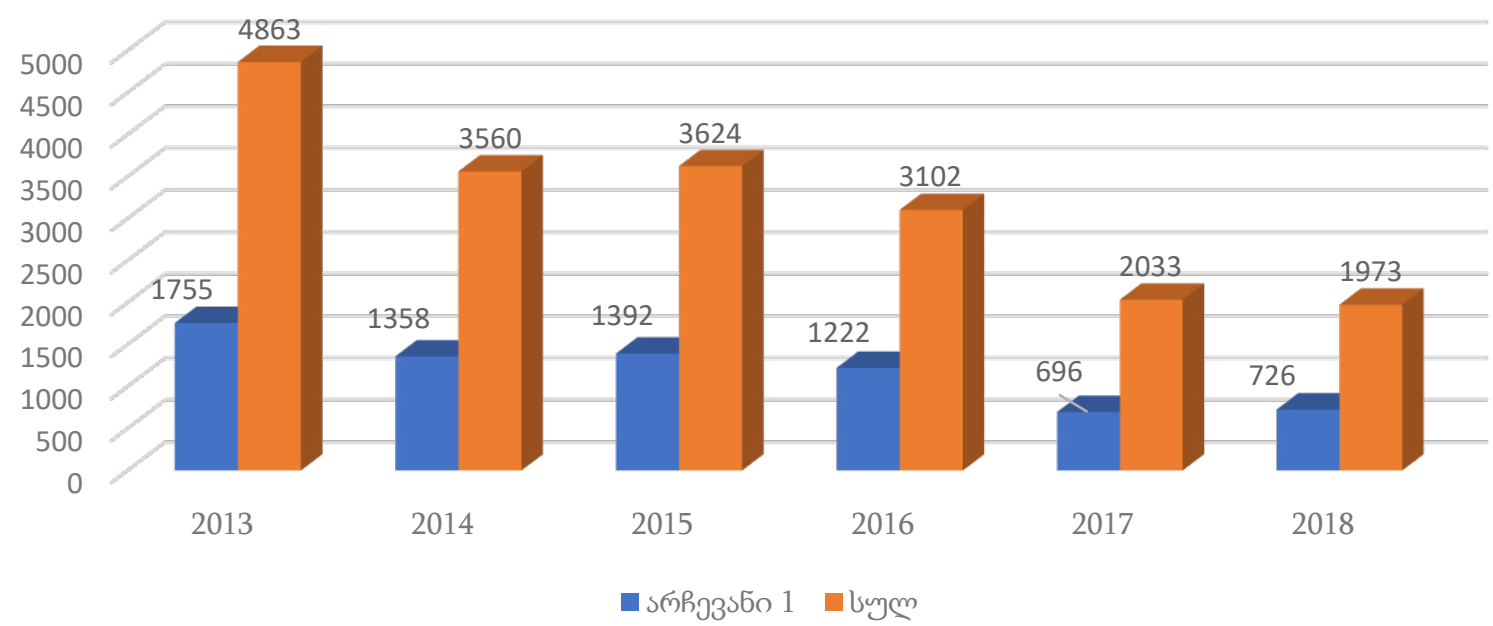




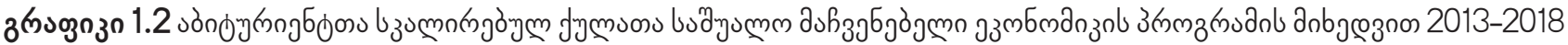
fimgònb anbjeznon (obly)

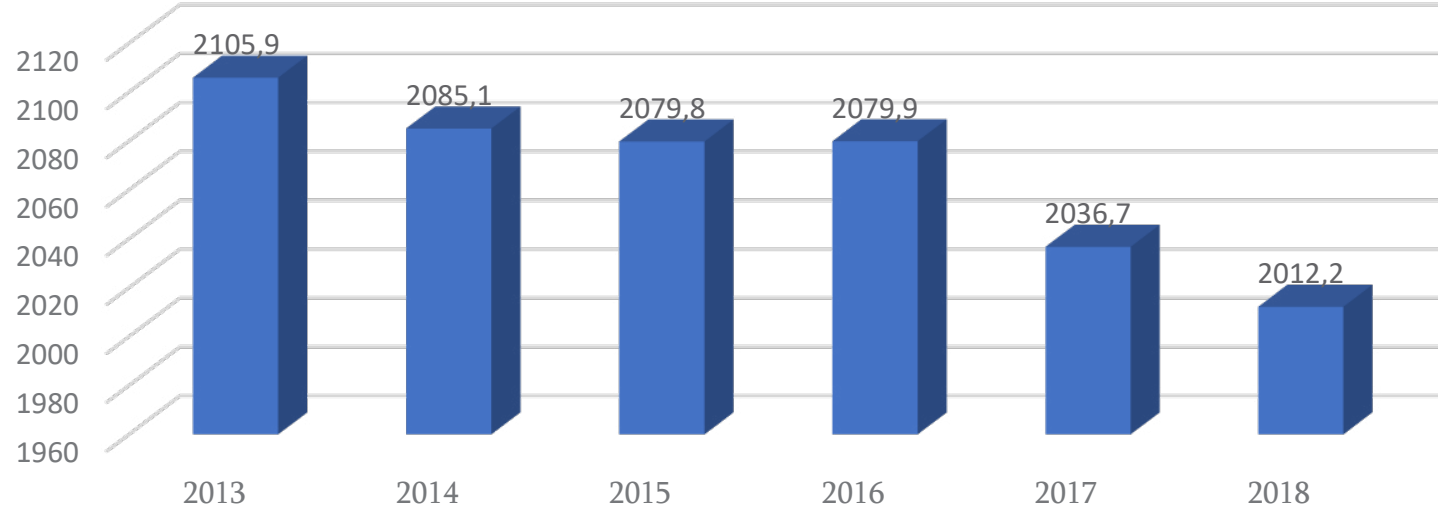

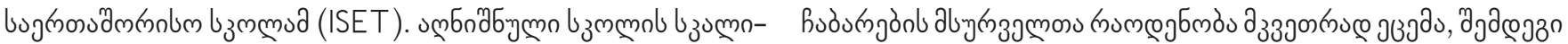

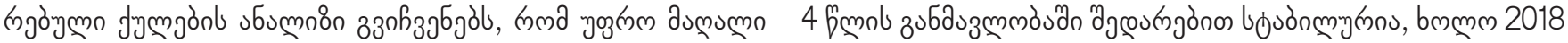

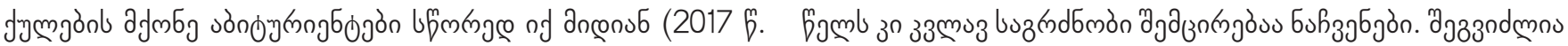
$-2135,8 ; 2018$ f. - 2110,1).

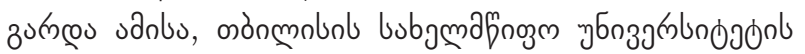

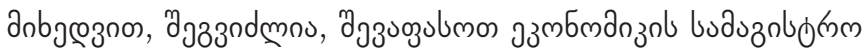

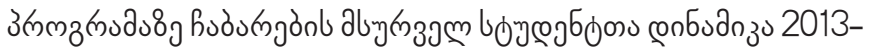

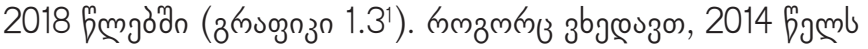

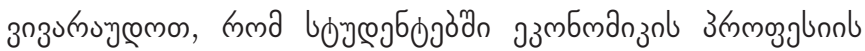

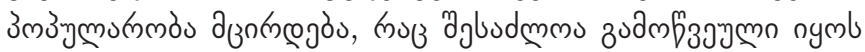

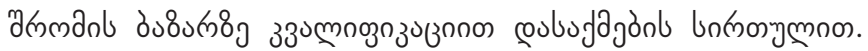

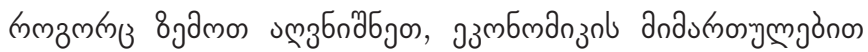

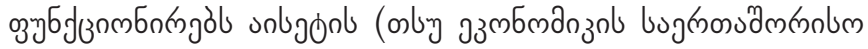

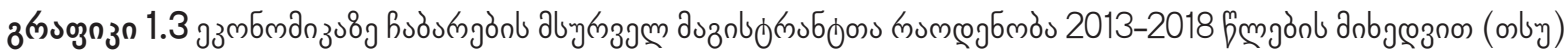

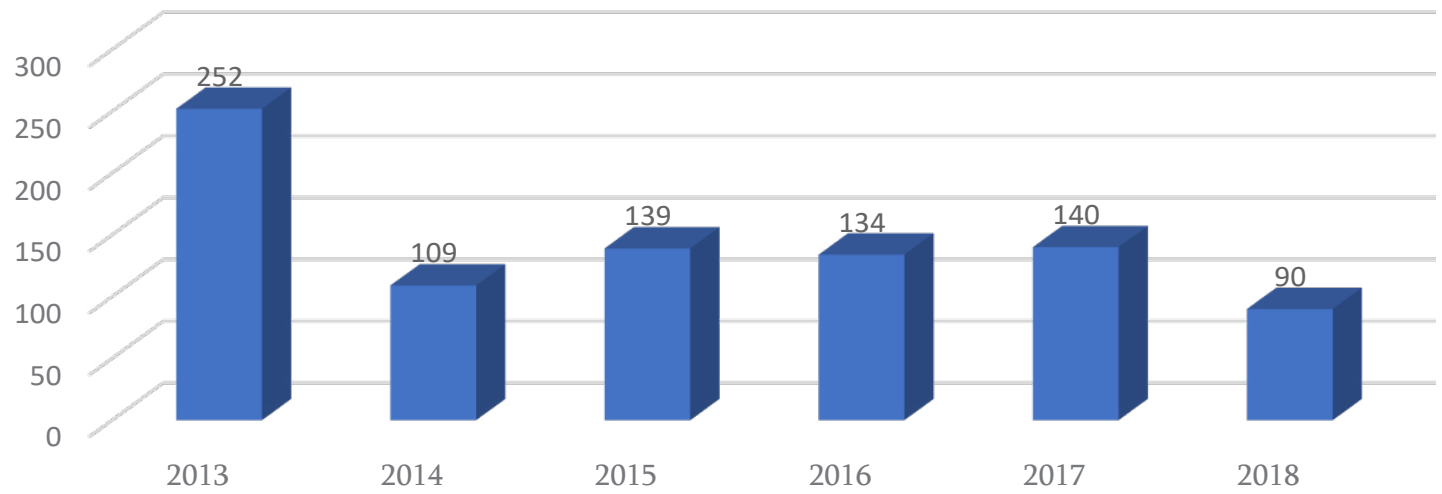

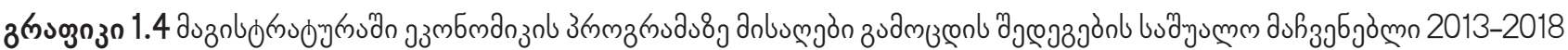

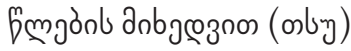

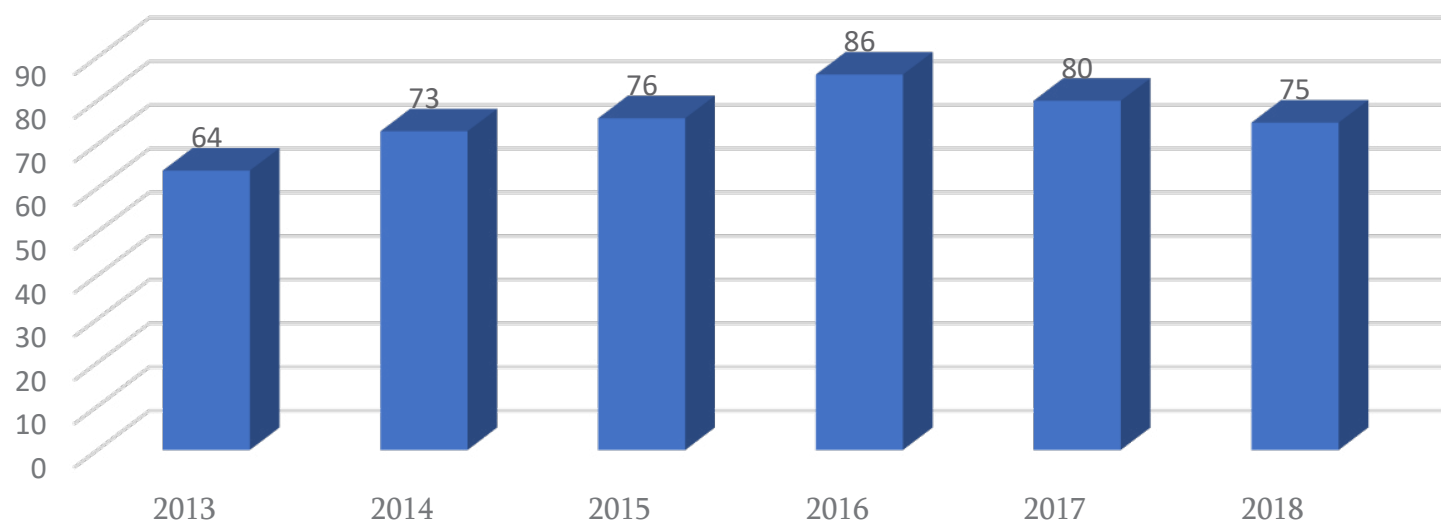

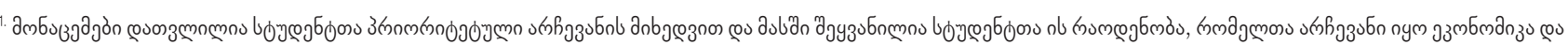

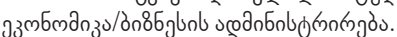




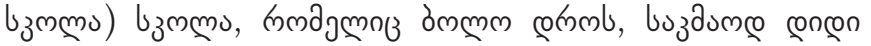

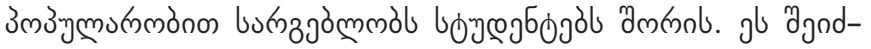

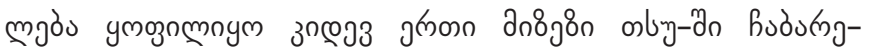

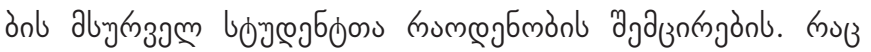

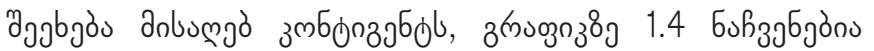

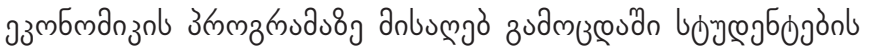

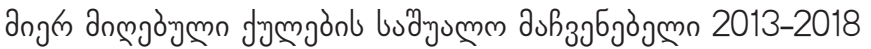

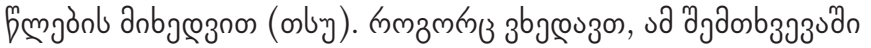

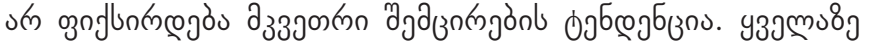

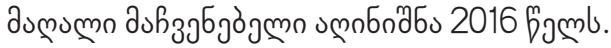

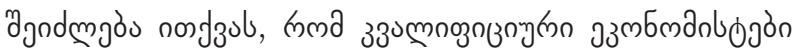

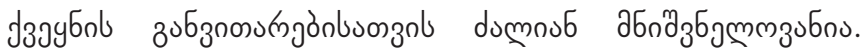

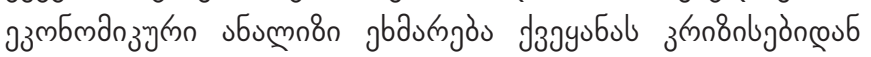
mszol cou

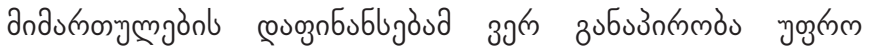

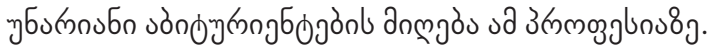

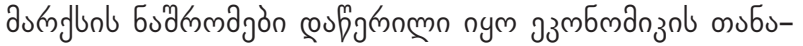

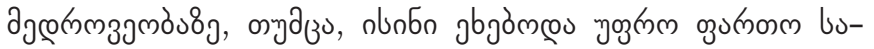

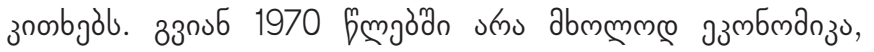

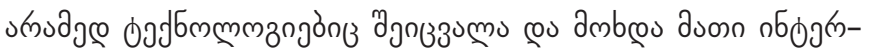

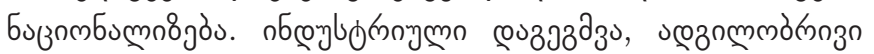

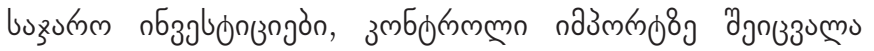

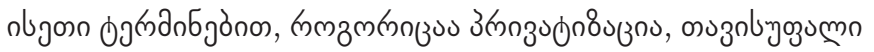

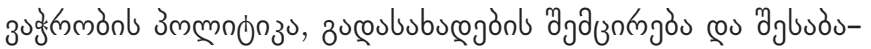

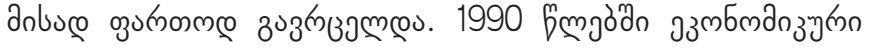

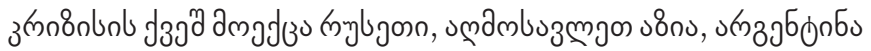

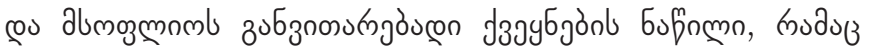

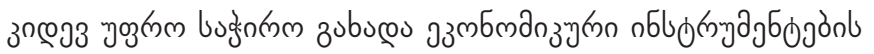

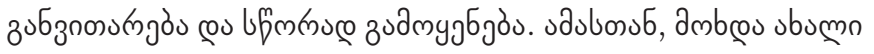

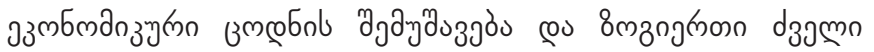

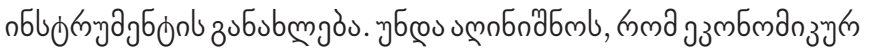

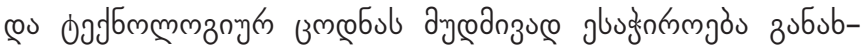

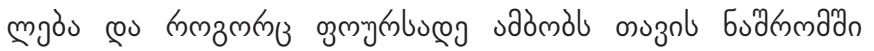

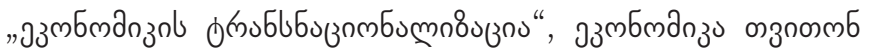

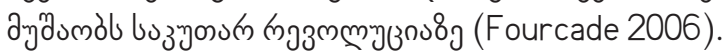

оुмचु

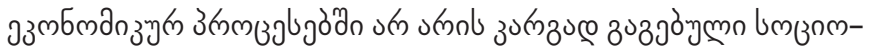

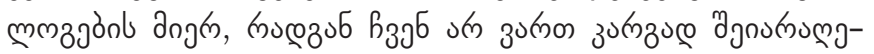

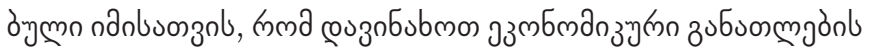
conougronl conbuanzu (Fourcade 2006).

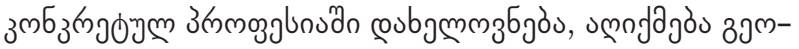

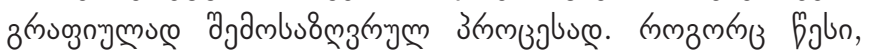

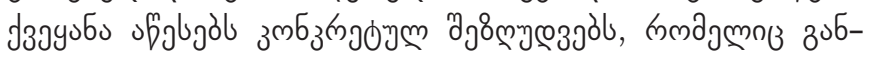

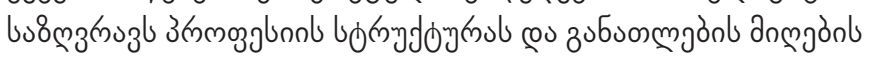

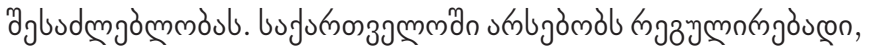
aymmon

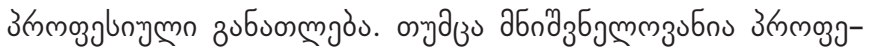

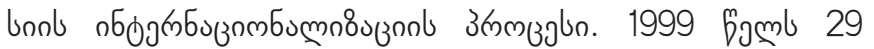

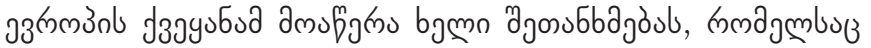

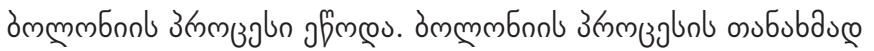

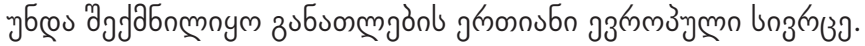

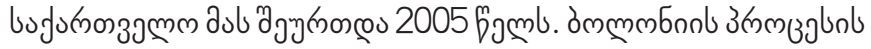

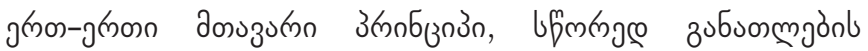

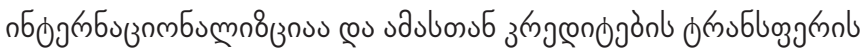

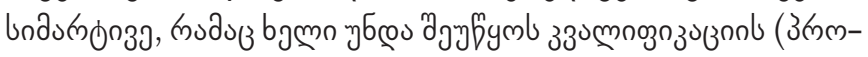

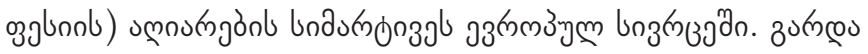

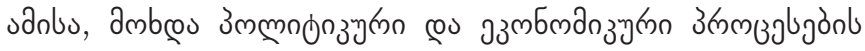

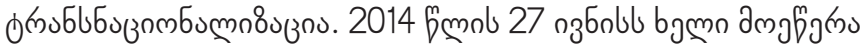

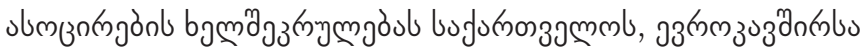

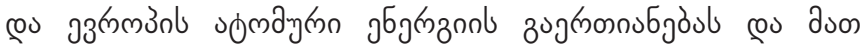

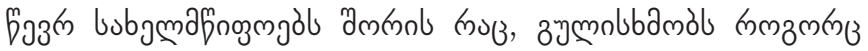

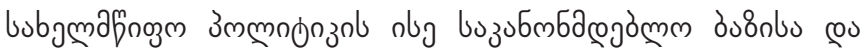

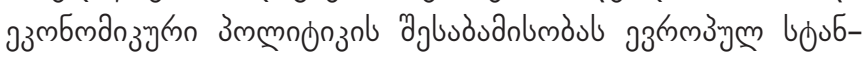

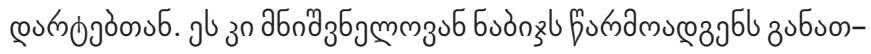

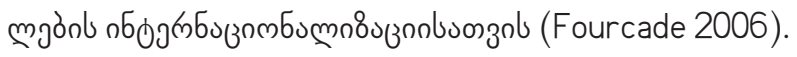

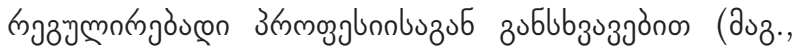

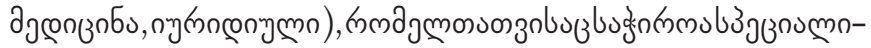

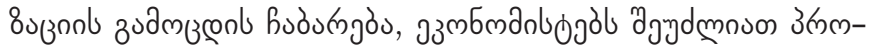

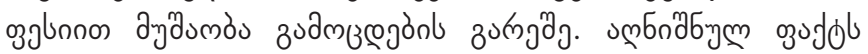
оुмचु

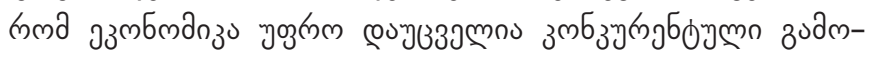

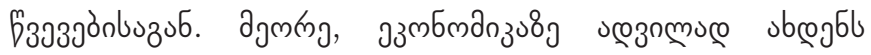

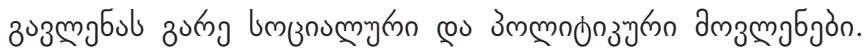

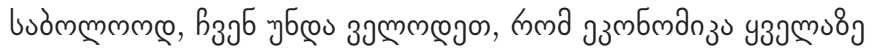

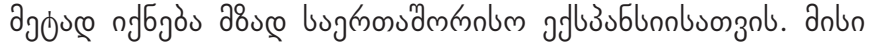

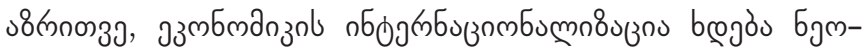

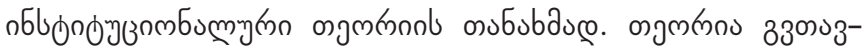

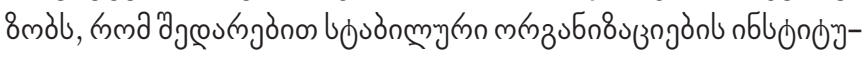

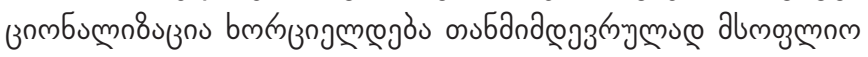
dubajuónon (Fourcade 2006).

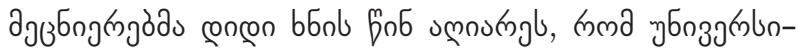

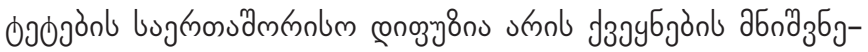

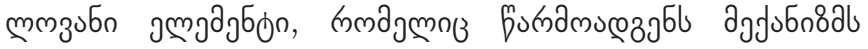

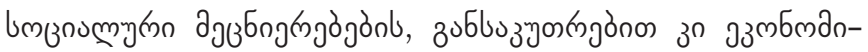

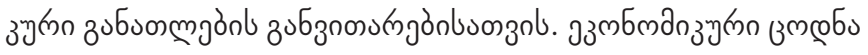

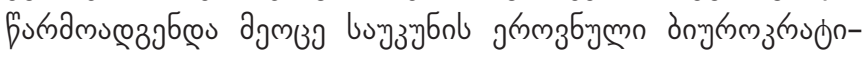

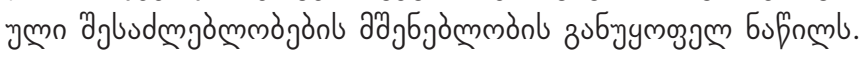

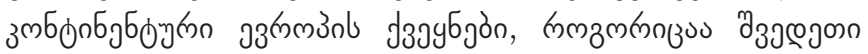

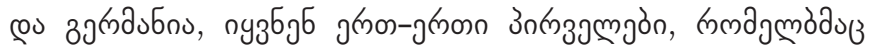

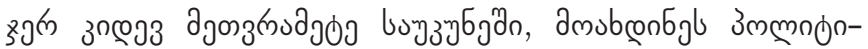

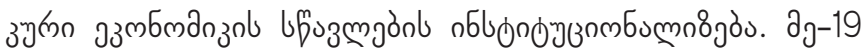

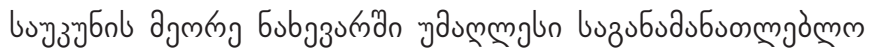

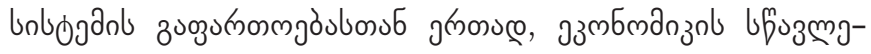

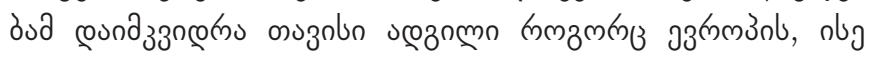

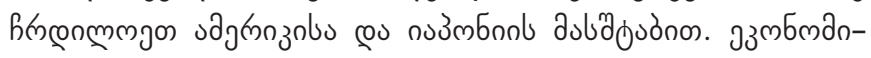

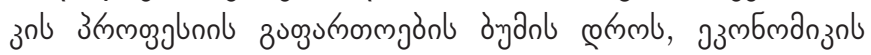

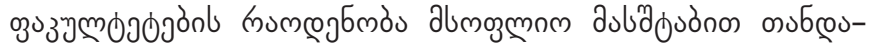

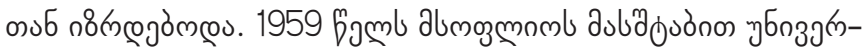

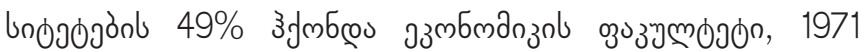

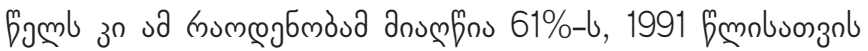




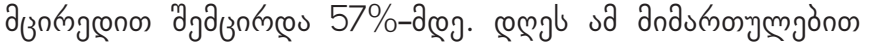

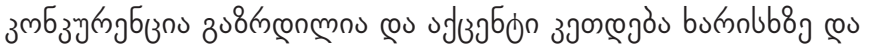

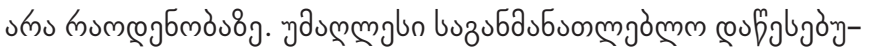

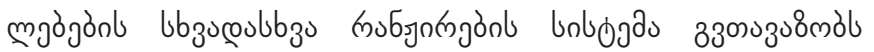
चб

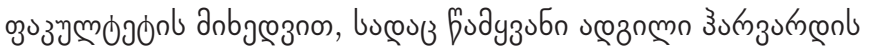

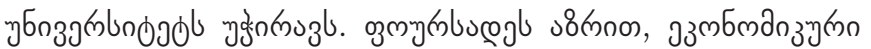

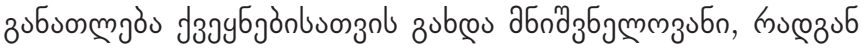

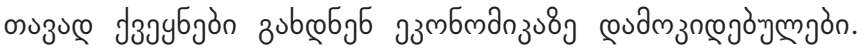

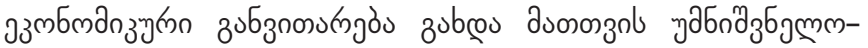

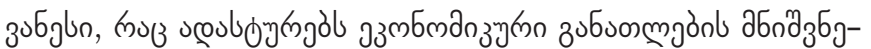

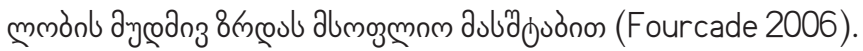

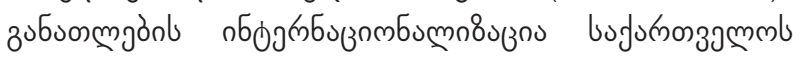

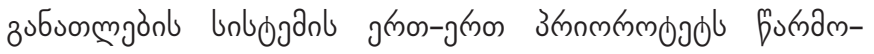

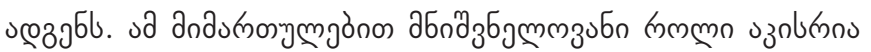

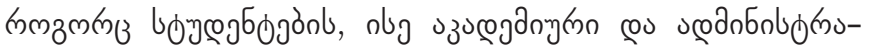

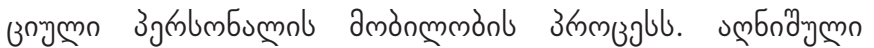

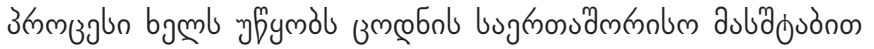

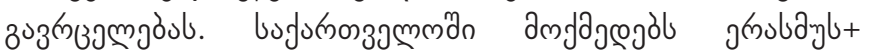

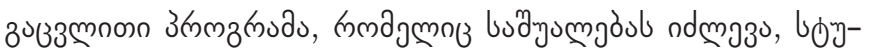

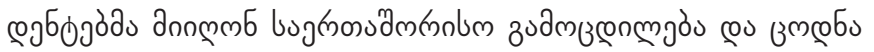

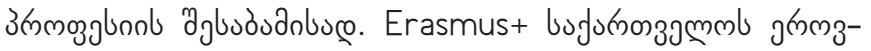

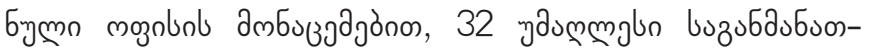

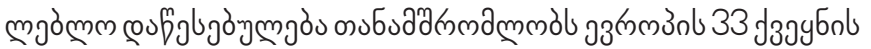

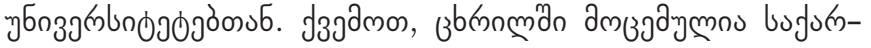

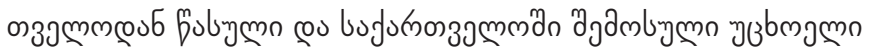

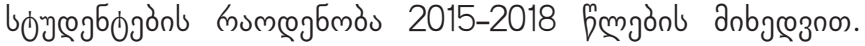

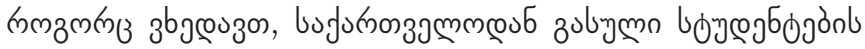

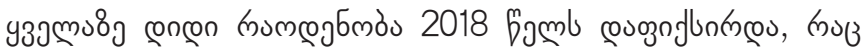

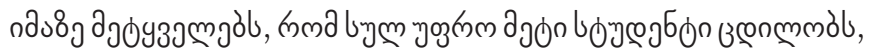

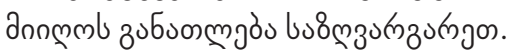

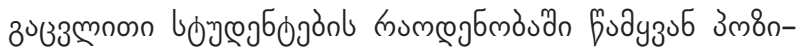

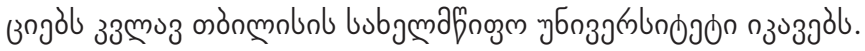

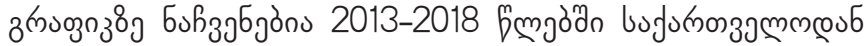

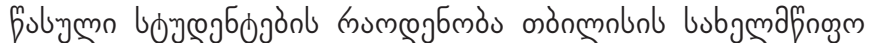

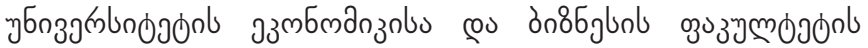

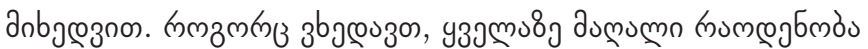

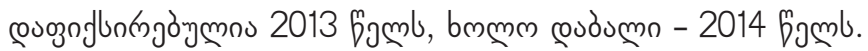

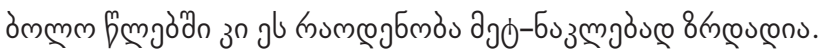

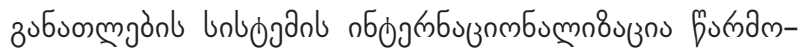
دезбаб

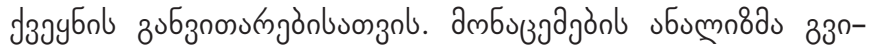

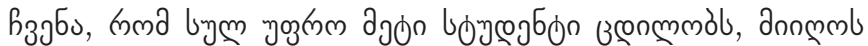

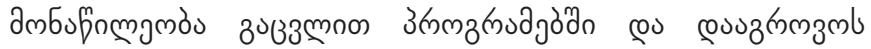

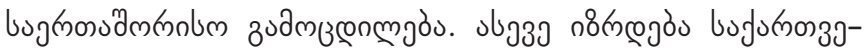

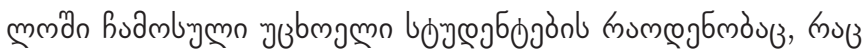

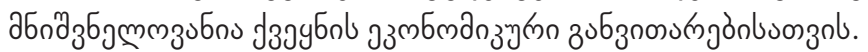

\section{cos336s}

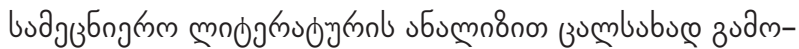

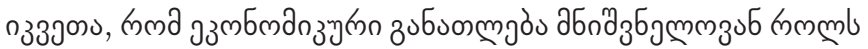

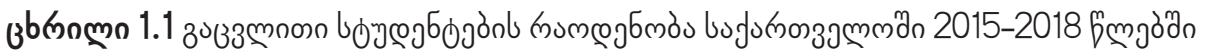

\begin{tabular}{|c|c|c|c|}
\hline бэмо & 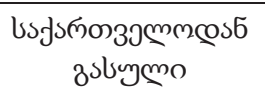 & 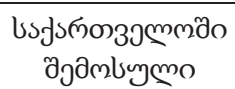 & bygm \\
\hline 2015 & 695 & 190 & 885 \\
\hline 2016 & 989 & 570 & 1559 \\
\hline 2017 & 818 & 515 & 1333 \\
\hline 2018 & 1105 & 697 & 1802 \\
\hline x̧ao & 3607 & 1972 & 5579 \\
\hline
\end{tabular}

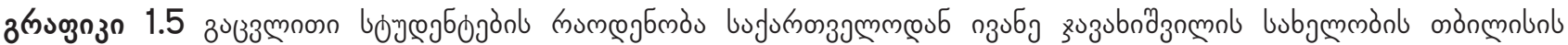

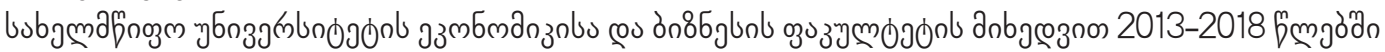

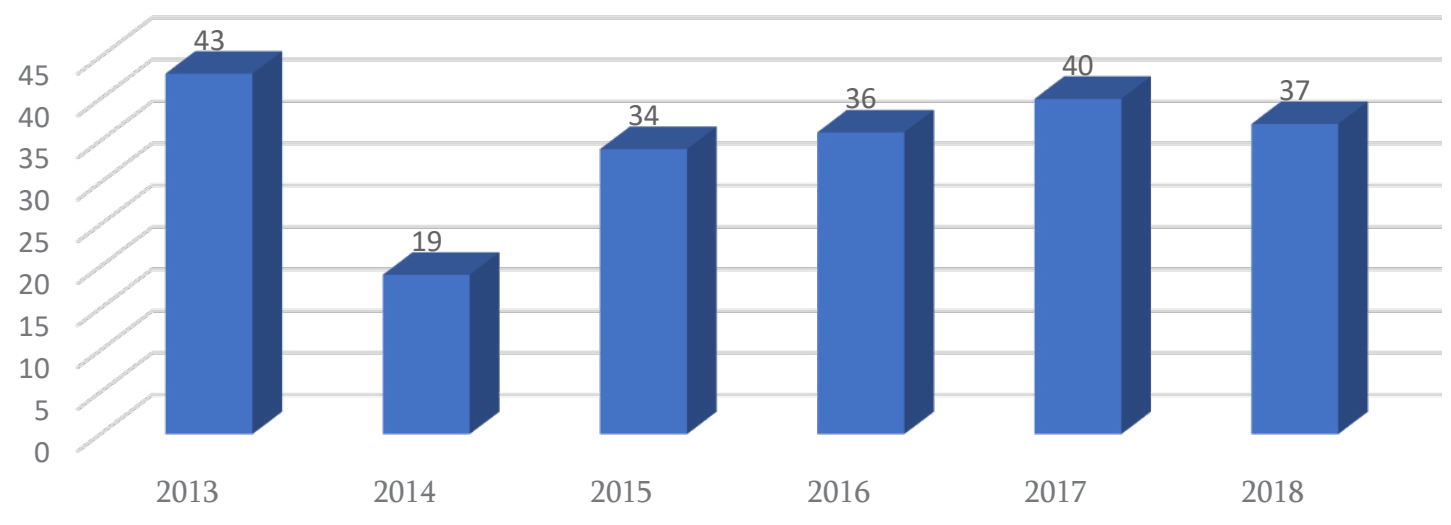




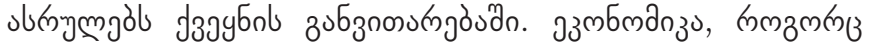

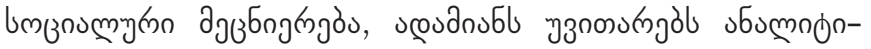

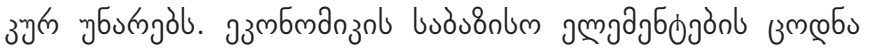

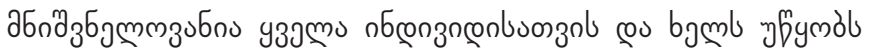

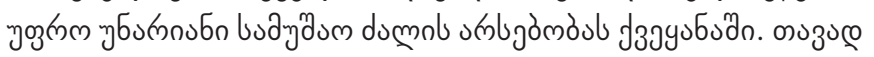

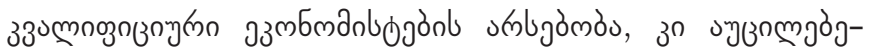

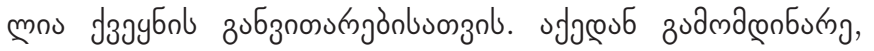

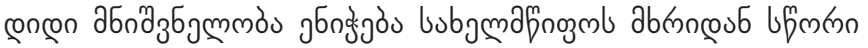
उmmnonzol zodungasul.

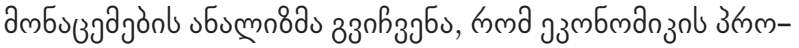

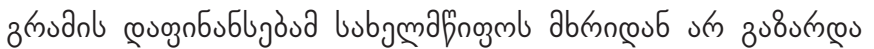

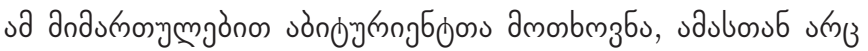

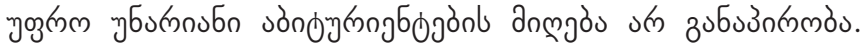

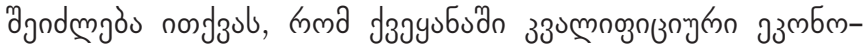

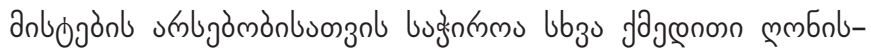

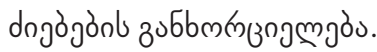

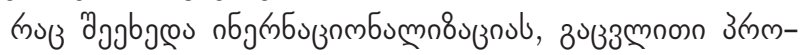

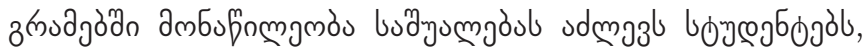

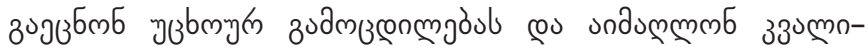

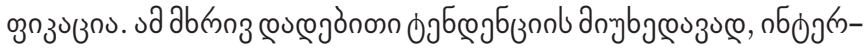

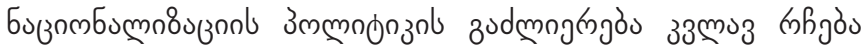

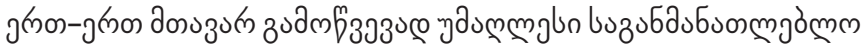
cosfjbjò̀ymgàjònluonzol.

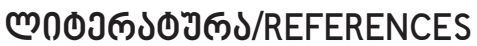

Arevadze, L. (2016). Effectiveness of fully state-funded faculties. Tbilisi (In Georgian). https://idfi.ge/ge/effectiveness-of-fullyfunded-faculties (03.01.2019).

Boddy, F. M. (1962). The Demand for Economists. The American Economic Review 52:2 (May): 503-508.

Boulding, K. E. (1969). Economic Education: The Stepchild too Is Father of the Man. The Journal of Economic Education 1 (Autumn): 7-11.

Fourcade, M. (2006). The Construction of a Global Profession: The Transnationalization of Economics. American Journal of Sociology 112:1 (July): 145-194.

Gunes, S. (2012). Economic Education in Industrial Design. Procedia - Social and Behavioral Sciences 47: 1352-1357

Keiser, N. F. (1956). The Role of Economics in General Education. The Journal of General Education 3 (April): 179-187.

Jacobson, M.\&Wilensky,U. (2009). Complex Systems in Education: Scientific and Educational Importance and Implications for the Learning Sciences. The Journal of the Learning Sciences 15 (Nov.): 11-34.

Peterson, J. (2013). Economics Education after the Crisis: Pluralism, History, and Institutions. Journal of Economic Issues 47:2: 401-410.

Robinson, W.\&Davis, James E. (1999). Technology, The Economics Profession, and Pre-College Economic Education. The Journal of Education Vol. 181, No. 3: 77-90. 